\title{
Buffer-aided Successive Opportunistic Relaying with Inter-Relay Interference Cancellation
}

\author{
Nikolaos Nomikos, Themistoklis Charalambous, Ioannis Krikidis, Dimitrios N. Skoutas, \\ Demosthenes Vouyioukas and Mikael Johansson
}

\begin{abstract}
In this paper we consider a simple cooperative network consisting of a source, a destination and a cluster of decode-and-forward relays characterized by the half-duplex constraint. At each time-slot the source and (possibly) one of the relays transmit a packet to another relay and the destination, respectively. When the source and a relay transmit simultaneously, inter-relay interference is introduced at the receiving relay. In this work, with the aid of buffers at the relays, we mitigate the detrimental effect of inter-relay interference through either interference cancellation or mitigation. More specifically, we propose the min - power opportunistic relaying protocol that minimizes the total energy expenditure per time slot under an inter-relay interference cancellation scheme. The min - power relay-pair selection scheme, apart from minimizing the energy expenditure, also provides better throughput and lower outage probability than existing works in the literature. The performance of the proposed scheme is demonstrated via illustrative examples and simulations in terms of outage probability and average throughput.
\end{abstract}

\section{INTRODUCTION}

Relaying cooperation is an efficient technique to combat fading and path-loss effects in wireless systems. It enables multiple nodes to create virtual multiple-input multiple-output (MIMO) configurations in order to provide transmit and/or receive spatial diversity to single-antenna destinations [1]. Traditional cooperative systems are characterized by the halfduplex constraint and thus relay nodes cannot receive and transmit data simultaneously resulting in bandwidth loss. In order to overcome this limitation, several techniques have been proposed in the literature [2]. Among them, the successive relaying scheme in [3] incorporates two relays and proposes a transmission overlap (source-relay (SR), relay-destination (RD)) which mimics full-duplex transmission. On the other hand, for networks with multiple relay nodes, relay selection has been introduced as a promising solution that exploits the available channel diversity degrees by keeping the implementation complexity low. In earlier works, relays were assumed to lack data buffers and selection was based on the maxmin criterion and its variations (see, for example, [4]-[6] and references therein). As a result, the relay that received the

N. Nomikos, D. Vouyioukas and D. N. Skoutas are with the Department of Information and Communication Systems Engineering, University of the Aegean, Karlovassi 83200, Samos, Greece (E-mails: \{nnomikos, dvouyiou, d. skoutas\} @aegean.gr).

T. Charalambous and M. Johansson are with the Automatic Control Lab, Electrical Engineering Department and ACCESS Linnaeus Center, Royal Institute of Technology (KTH), Stockholm, Sweden (Emails: \{themisc, mikaelj\}@kth.se).

I. Krikidis is with the Department of Electrical and Computer Engineering, University of Cyprus, Nicosia 1678 (E-mail: krikidis@ucy.ac.cy). source signal was the same with the one that forwarded the signal towards the destination.

In the recent adoption of buffer-aided relays this coupling is broken. Ikhlef et al. [8] propose a novel criterion based on the max - max relay selection (MMRS), in which the relay with the best SR link is selected for reception and the relay with the best RD link is selected for transmission. Another work that adopts the MMRS is the one in [9] which aims to recover the half-duplex loss of relaying by having different relays selected in the same time slot to receive and transmit. Krikidis et al. [10] proposed the max - link protocol which allows for all the SR and RD links to enter the competition for the best one through which a signal will be transmitted, thus providing additional freedom in the scheduled transmissions in each time slot. In [11] buffer-aided successive opportunistic relay selection (BA-SOR) was proposed where buffering allowed outage probability and capacity increase through joint relaypair selection.

In this work, a buffer-aided successive opportunistic relaying protocol is proposed with inter-relay interference (IRI) cancellation. In contrast to other works in the literature, we consider the arising IRI and we mitigate its detrimental effect through interference cancellation. More specifically, we propose the $\min$ - power relay-pair selection policy that acts in conjunction with interference cancellation and adjusts accordingly the power levels required to support the end-toend communication. The contribution of this work is twofold: (i) Buffer-aided relays and interference cancellation are combined for the first time, decoupling the necessity of the receiving relay to transmit in the next time slot, even if the channel is in outage.

(ii) Power adjustment is also included in our scheme. In this way, the total energy expenditure in the network is minimized, as well as the inter-relay interference, thus reducing the outage probability of the network.

The structure of the remainder of this paper is as follows. In Section II, we present the system model. In Section III, the min - power relay-pair selection policy is described in detail. Then, a model of this communication scheme and an outage probability analysis is performed in Section IV, while numerical results are provided in Section V. Finally, conclusions and future directions are discussed in Section VI.

\section{SYSTEM MODEL}

We assume a simple cooperative network consisting of one source $S$, one destination $D$ and a cluster $\mathcal{C}$ with $K$ decode- 
and-forward (DF) half-duplex relays $R_{k} \in \mathcal{C}(1 \leq k \leq K)$. A direct link between the source and the destination does not exist and communication can be established only via relays [4]. Each relay $R_{k}$ holds a buffer (data queue) $Q_{k}$ of capacity $L$ (number of data elements) where it can store source's data that have been decoded at the relay and can be forwarded to the destination. At the beginning of the transmission each relay buffer is empty and the parameter $l_{k} \in \mathbb{Z}_{+}, l_{k} \in[0, L]$ denotes the number of data elements that are stored in buffer $Q_{k}$. When a relay is activated for reception its data element number is increased in accordance with the rate that the source is transmitting, denoted as $r_{0}$ bits per channel use (BPCU), while the relay selected for transmission decreases its data element number by $r_{0}$, since fixed rate transmission is assumed. We denote by $\mathcal{T}$ all the relays for which their buffer is not empty, i.e., $\mathcal{T}=\left\{R_{k}: l_{k}>0\right\}$. Set $\mathcal{T}$ is included in the set of all the relays, hence $\mathcal{T} \subseteq \mathcal{C}$.

Time is considered to be slotted and at each time-slot the source $S$ and (possibly) one of the relays $R_{k}$ transmit with power $P_{S}$ and $P_{R_{k}}$, respectively. The source is assumed to be saturated (it has always data to transmit) and the information rate is equal to $r_{0}$. The retransmission process is based on an acknowledgment/negative-acknowledgment (ACK/NACK) mechanism, in which short-length error-free packets are broadcasted by the receivers (either a relay $R_{k}$ or the destination $D$ ) over a separate narrow-band channel in order to inform the network of that packet's reception status.

All wireless links exhibit fading and additive white Gaussian noise (AWGN). The fading is assumed to be stationary, with frequency non-selective Rayleigh block fading. This means that the fading coefficients $h_{i j}$ (for the $i \rightarrow j$ link) remain constant during one slot, but change independently from one slot to another according to a circularly symmetric complex Gaussian distribution with zero mean and unit variance. The channel gains are $g_{i j}=\left|h_{i j}\right|^{2}$ and exponentially distributed, taking values in the range $\left(0, \sigma_{i j}^{2}\right)$. The power level chosen by transmitter $i$ is denoted by $P_{i} . n_{i}$ denotes the variance of thermal noise at the receiver $i$, which is assumed to be AWGN.

Since we implement successive relaying whenever possible, we (may) have concurrent transmissions by the source and one relay, taking place at the same time slot. This relaying scheme requires at least two relays, as one relay receives the source's frame while another relay is forwarding a previous frame to the destination, thus recovering the half-duplex loss of regular relays, since the destination receives one frame per transmission phase with the exception of the first phase. However, the overlapping transmissions result in IRI and the source has to consider the interference power that the relay chosen to receive the source's signal, denoted by $R_{k}$, is experiencing by the relay chosen to transmit a signal to the destination, denoted by $R_{t}$. Since our focus is to investigate the performance of a new buffer-aided successive opportunistic relay selection scheme under a global channel state information (CSI) assumption, the implementation issues (i.e., distributed implementations [4], CSI acquisition, etc.) are beyond the scope of this work. However, a centralized

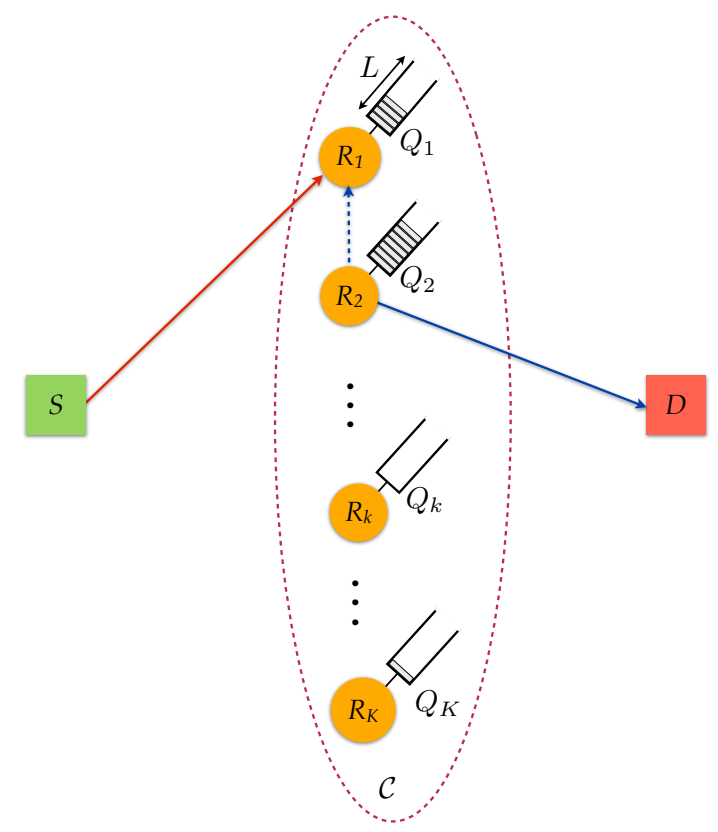

Fig. 1. The system model: Source $S$ communicates with destination $D$ via a cluster of relays $R_{k} \in \mathcal{C}, k \in[1, K]$.

approach is assumed each time the source selects the receiving and transmitting relays. This requires a brief initialization process prior to each transmission phase in order for the CSI to be fed back to the source. Compared to other state-of-the-art works such as [9], an additional overhead is introduced due to the acquisition of the inter-relay links' CSI. However, this loss can be recovered through the possibility of interference cancellation due to the optimal relay-pair selection.

\section{III. min - power RELAY SELECTION POLICY}

In this section, we present the relay selection policy, called min - power. This relay selection scheme is associated with a one-slot cooperative protocol (similar to the max - link relay selection policy [10]), rather than two-slot protocols (followed in many relay selection protocols [7]-[9]). At each time slot, the source $S$ transmits data to a selected relay (denoted by $R_{r} \in \mathcal{K}$ ) with a non-full buffer, and another relay with a nonempty buffer (denoted by $R_{t} \in \mathcal{T}, R_{t} \neq R_{r}$ ) transmits data to the destination $D$.

A packet is successfully transmitted from the transmitting relay $R_{t}$ to the destination $D$ if $\operatorname{SNR}_{R_{t} D} \geq \gamma_{0}$, i.e.,

$$
\frac{g_{R_{t} D} P_{R_{t}}}{n_{D}} \geq \gamma_{0}, \quad R_{t} \in \mathcal{T}, R_{t} \neq R_{r} .
$$

A packet is successfully transmitted from source $S$ to the receiving relay $R_{r}$, if $\operatorname{SINR}_{S R_{r}} \geq \gamma_{0}$, i.e.,

$$
\frac{g_{S R_{r}} P_{S}}{g_{R_{t} R_{r}} P_{R_{t}} \mathbb{I}\left(R_{t} R_{r}\right)+n_{R_{r}}} \geq \gamma_{0}, \quad R_{r} \in \mathcal{C}, R_{r} \neq R_{t},
$$

where $\mathbb{I}\left(R_{t} R_{r}\right)$ is a factor indicating whether interference cancellation is satisfied and it is described by

$$
\mathbb{I}\left(R_{t} R_{r}\right)= \begin{cases}0, & \text { if } \frac{g_{R_{t} R_{r}} P_{R_{t}}}{g_{S R_{r}} P_{S}+n_{R_{r}}} \geq \gamma_{0}, \\ 1, & \text { otherwise. }\end{cases}
$$


The following proposition states that if the maximum powers $P_{S}^{\max }$ and $P_{R_{t}}^{\max }$ are large enough (thus not imposing any limitations/constraints), for each pair of relays $R_{r}$ and $R_{t}$, then we can always find power levels such that interference cancellation conditions are satisfied. When interference cancellation conditions hold, the interfering signal is subtracted at the relay prior to the decoding of the source signal.

Proposition 1. Let $P_{S}^{\max }=\infty$ and $P_{R_{t}}^{\max }=\infty$. For each pair of relays $R_{r}$ and $R_{t}$, there exist $P_{S}$ and $P_{R_{t}}$ such that $\mathbb{I}\left(R_{t} R_{r}\right)=0, \operatorname{SNR}_{R_{t} D} \geq \gamma_{0}$ and $\operatorname{SINR}_{S R_{r}} \geq \gamma_{0}$. The minimum power levels $P_{S}^{*}$ and $P_{R_{t}}^{*}$ are achieved when $S N R_{R_{t} D}=\operatorname{SINR}_{S R_{r}}=\gamma_{0}$, and are given by

$$
\begin{aligned}
P_{S}^{*} & =\frac{\gamma_{0} n_{R_{r}}}{g_{S R_{r}}}, \\
P_{R_{t}}^{*} & =\max \left\{\frac{\gamma_{0} n_{D}}{g_{R_{t} D}}, \frac{n_{R_{r}} \gamma_{0}\left(\gamma_{0}+1\right)}{g_{R_{t} R_{r}}}\right\} .
\end{aligned}
$$

Proof: For IC to take place, according to (3), we have

$$
g_{R_{t} R_{r}} P_{R_{t}} \geq \gamma_{0}\left(g_{S R_{r}} P_{S}+n_{R_{r}}\right)
$$

Given that $P_{R_{t}}$ is chosen such that (5) is fulfilled, then (2) becomes

$$
\frac{g_{S R_{r}} P_{S}}{n_{R_{r}}} \geq \gamma_{0},
$$

and since $P_{S}$ does not depend on $P_{R_{t}}$, the minimum power of $S$ is given with equality; i.e.,

$$
P_{S}^{*}=\frac{\gamma_{0} n_{R_{r}}}{g_{S R_{r}}} .
$$

Substituting (4a) into (5) we have $g_{R_{t} R_{r}} P_{R_{t}} \geq n_{R_{r}} \gamma_{0}\left(\gamma_{0}+1\right)$. Hence, the minimum $P_{R_{t}}$ is given by

$$
P_{R_{t}}^{*}=\max \left\{\frac{\gamma_{0} n_{D}}{g_{R_{t} D}}, \frac{n_{R_{r}} \gamma_{0}\left(\gamma_{0}+1\right)}{g_{R_{t} R_{r}}}\right\} .
$$

Proposition 1 provides the minimum power levels of $S$ and $R_{t}$, provided that their maximum power levels do not impose any constrain and hence the IC conditions are satisfied. In the next proposition, we find the conditions under which IC cannot take place and we find the optimal power levels of $S$ and $R_{t}$.

Proposition 2. For each pair of relays $R_{r}$ and $R_{t}$, interference cancellation is feasible if and only if

$$
P_{R_{t}}^{*}=\max \left\{\frac{\gamma_{0} n_{D}}{g_{R_{t} D}}, \frac{n_{R_{r}} \gamma_{0}\left(\gamma_{0}+1\right)}{g_{R_{t} R_{r}}}\right\} .
$$

When interference cancellation is infeasible, the signal from $R_{t}$ can be decoded successfully at $D$ if and only if

$$
\frac{\gamma_{0} n_{D}}{g_{R_{t} D}} \leq P_{R_{t}}^{\max }
$$

In addition, the signal from $S$ can be decoded successfully at $R_{r}$ if and only if

$$
\gamma_{0}\left(\frac{g_{R_{t} R_{r}}}{g_{S R_{r}}} P_{R_{t}}+\frac{n_{R_{r}}}{g_{S R_{r}}}\right) \leq P_{S}^{\max } .
$$

When (6), (7) and (8) hold, the minimum power levels $P_{S}^{\dagger}$ and
$P_{R_{t}}^{\dagger}$ are achieved when $S N R_{R_{t} D}=S I N R_{S R_{r}}=\gamma_{0}$, and are given by

$$
\begin{aligned}
P_{S}^{\dagger} & =\gamma_{0}\left(\frac{g_{R_{t} R_{r}}}{g_{S R_{r}}} P_{R_{t}}+\frac{n_{R_{r}}}{g_{S R_{r}}}\right) \\
P_{R_{t}}^{\dagger} & =\frac{\gamma_{0} n_{D}}{g_{R_{t} D}} .
\end{aligned}
$$

Proof: Interference cancellation cannot take place when the maximum power of $R_{t}$ is not high enough, such that its signal can be decoded by $R_{r}$, i.e.,

$$
\frac{g_{R_{t} R_{r}} P_{R_{t}}^{\max }}{g_{S R_{r}} P_{S}+n_{R_{r}}}<\gamma_{0} .
$$

but it should definitely be high enough to be decoded at the destination, i.e.,

$$
\frac{\gamma_{0} n_{D}}{g_{R_{t} D}} \leq P_{R_{t}} \leq P_{R_{t}}^{\max }
$$

Then, $P_{S}$ should be high enough, so that the transmitting signal can be successfully decoded at $R_{r}$. Hence,

$$
\gamma_{0}\left(\frac{g_{R_{t} R_{r}}}{g_{S R_{r}}} P_{R_{t}}+\frac{n_{R_{r}}}{g_{S R_{r}}}\right) \leq P_{S} \leq P_{S}^{\max }
$$

Substituting (11) into (12) we have

$$
\gamma_{0}\left(\frac{g_{R_{t} R_{r}}}{g_{S R_{r}}} \frac{\gamma_{0} n_{D}}{g_{R_{t} D}}+\frac{n_{R_{r}}}{g_{S R_{r}}}\right) \leq P_{S} \leq P_{S}^{\max } .
$$

Making use of Propositions 1 and 2 we will now describe the min - power relay selection algorithm. The algorithm is implemented in the following steps:

1) First, for each possible pair of relays, we carry an $I C$ feasibility check, i.e., we check through (6) if interference cancellation is feasible.

2) If IC is feasible, then

(i) assuming IC took place, $P_{R_{t}}^{*}$ and $P_{S}^{*}$ are as given in (4b) and (4a), respectively. Note that since the feasibility criterion is satisfied, then $P_{R_{t}}^{*} \leq P_{R_{t}}^{\max }$. Nevertheless, we need to check separately whether $P_{S}^{*} \leq P_{S}^{\max }$.

(ii) assuming IC did not take place, $P_{R_{t}}^{\dagger}$ and $P_{S}^{\dagger}$ are as given in (9b) and (9a), respectively. $P_{R_{t}}^{\dagger} \leq P_{R_{t}}^{\max }$ is satisfied by the feasibility check, but we need again to check separately whether $P_{S}^{\dagger} \leq P_{S}^{\max }$.

(iii) The minimum energy expenditure at a specific time slot for each pair is the minimum sum of the powers for the two cases, i.e., $\min \left\{P_{S}^{*}+P_{R_{t}}^{*}, P_{S}^{\dagger}+P_{R_{t}}^{\dagger}\right\}$. Note that if IC can take place, this might require $P_{R_{t}}^{*}>P_{R_{t}}^{\dagger}$, such that $P_{S}^{*}+P_{R_{t}}^{*}>P_{S}^{\dagger}+P_{R_{t}}^{\dagger}$.

3) If IC is infeasible, then we use case (ii) only from step 2). 4) We compare the minimum energy expenditure for all possible relay pairs and we choose the minimum among them.

Remark 1. Note that in the worst case scenario (in which all the queues are neither empty nor full), there will be $K \times$ $(K-1)$ combinations. Hence, the worst case complexity of the problem is $\mathcal{O}\left(K^{2}\right)$. 


\section{MODEL AND OUTAGE PROBABILITY ANALYSIS}

In this section, the outage probability behavior of the min - power relay selection scheme follows the theoretical framework of [10], which is also a relay network with finite buffers. The main differentiation compared to [10] is that we have additional ways of transmission through successive relaying; in other words, we have additional links from a certain buffer state to others. This will be more clearly reflected in the illustrative example. Note that the possibility of having a successive transmission requires two links to offer a SINR at the receivers equal to or above $\gamma_{0}$, at the same time, otherwise transmission is based on single-link selection. So, an outage takes place when $\gamma_{0}$ can not be achieved even in a single-link transmission; thus, $\mathbb{P}_{\text {out }} \triangleq \mathbb{P}\left(\Gamma_{k}(\mathbf{P})<\gamma_{0}\right)$.

\section{A. Construction and properties of the state transition matrix of the $M C$}

We first formulate the state transition matrix of the Markov Chain (MC), denoted as $\mathbf{A}, \mathbf{A} \in \mathbb{R}^{(L+1)^{K} \times(L+1)^{K}}$. More specifically, $\mathbf{A}_{i, j}=\mathbb{P}\left(s_{i} \rightarrow s_{j}\right)=\mathbb{P}\left(X_{t+1}=s_{j} \mid X_{t}=s_{i}\right)$ are the transition probabilities to move from a state $s_{i}$ to a state $s_{j}$. The transition probability depends on the number of relays that are available for cooperation.

Remark 2. As we consider finite buffers, relays that have full buffers cannot compete in the selection of the best relay that will receive the source's signal. Also, relays with empty buffers are not able to transmit and as a result they are excluded from the best transmitting relay selection. Moreover, when there is no possibility of transmitting successively through two selected relays our system reduces to the max - link relay selection scheme. The number of links that are available in this mode is reduced if the relays have full or empty buffers.

It is easily shown that the state transition matrix $\mathbf{A}$ of the MC that models the buffer states is SIA (Stochastic Irreducible and Aperiodic). As a result, the MC has a stationary distribution, hereafter denoted as $\boldsymbol{\pi}$. Hence, we can explore how the data are being sent across the relays and the long-term share of the resources.

An outage event occurs when there is no change in the buffer status ${ }^{1}$; thus, the outage probability of the system is given by

$$
P_{\text {out }}=\sum_{i=1}^{(L+1)^{K}} \boldsymbol{\pi}_{i} \bar{p}_{D_{i}}=\operatorname{diag}(\mathbf{A}) \boldsymbol{\pi},
$$

where $D_{i}$ is the number of outgoing links. Here, the main idea of the outage probability analysis is outlined. The detailed analysis of this setup appears in a longer version in [?], and it will be presented in a journal version of this paper.

\footnotetext{
${ }^{1}$ Note that $\mathrm{min}$ - power first checks if a successive transmission is possible and if it fails due to one or both hops being in outage, then it operates as a single-link selection scheme. If single-link selection fails then there is no change in the buffer status and the system is in outage.
}

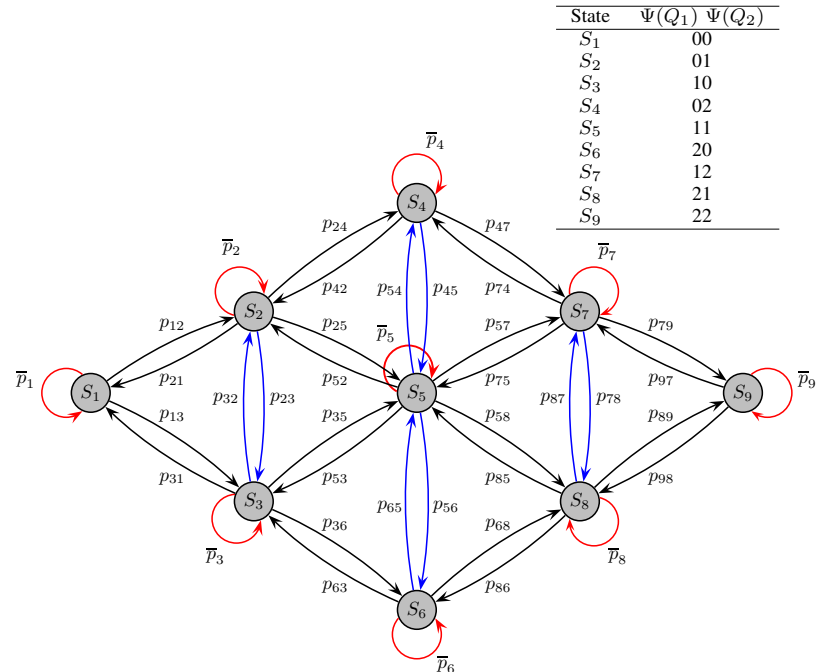

Fig. 2. State diagram of the Markov chain representing the states of the buffers and the transitions between them for a case with $K=2$ and $L=$ 2. Compared to the max - link scheme in [10], the min - power model includes extra transition states due to the successive nature of the protocol.

\section{B. Illustrative example with $K=2$ relays with $L=2$}

In what follows, we present an illustrative example that showcases the behavior of our approach for different parameters. Since we have a scheme that employs successive transmissions the simplest case is when two relays are available. Assuming that each relay has a buffer size equal to two, we show its state transition diagram in Fig.2, with the nine possible states for the buffers of the two relays.

The steady state of the system for different values of SNR can be found by using the method described in IV-A.

\section{NumERICAL RESUltS}

Here, we evaluate the min - power scheme in terms of: 1)outage probability and 2)average throughput. min - power is compared to best-relay selection (BRS) [4], successive opportunistic relaying (SOR) [12], hybrid relay selection ( $\max -\max )$ [8], $\max$ - link selection [10] and BA-SOR [11]. In addition, we provide a selection bound corresponding to the case where interference is negligible and all links are available for selection as in [9]. Also, the selection bound scheme is coupled with single-link transmissions when successive transmissions fail, in order to provide a fair comparison with min - power.

Outage Probability. Fig.3, illustrates the outage probability results; each scheme employs $K=2$ relays with buffer size $L=2$, while min - power is also depicted for additional buffer sizes $L=8$ and $L=100$. SOR has the worst performance since it lacks buffers and selection is coupled to the previous transmission phase, while BA-SOR improves on this metric due to buffering. Also, max - max offers 1.5 $\mathrm{dB}$ improvement over BRS due to buffering. Better results are achieved by max - link as outage performance is improved by almost $4 \mathrm{~dB}$ due to the flexible link selection. As min - power reduces to max - link when successive transmissions fail, we observe similar results between these two schemes. For $K=2$ and $L=2$, min - power exhibits a $0.5 \mathrm{~dB}$ gain for 


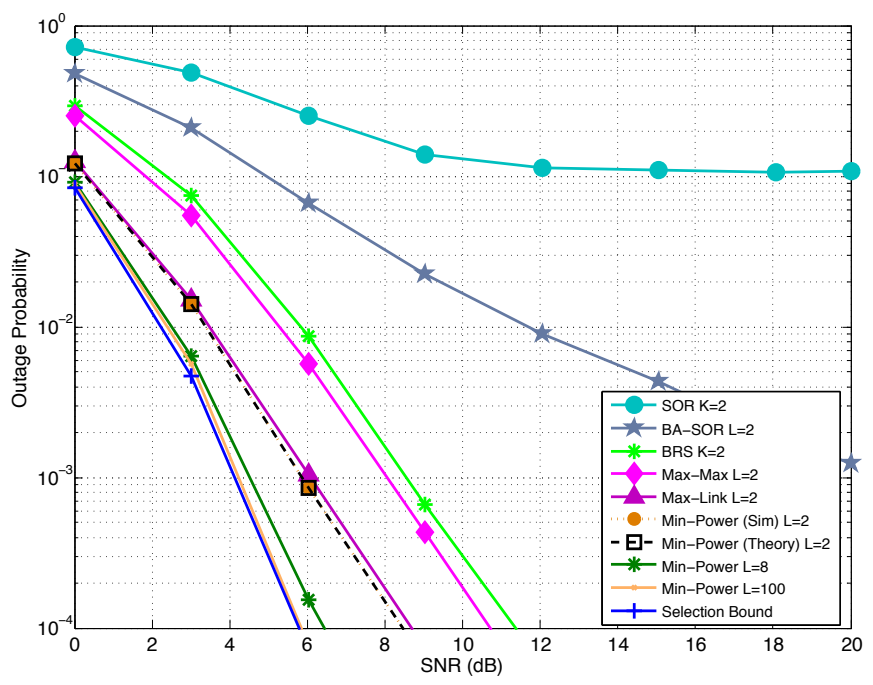

Fig. 3. Outage probability for increasing transmit SNR for $K=2$.

high SNR. The increased interconnection between buffer states guarantees that states $S_{1}(00)$ and $S_{9}$ (22) offering the least diversity, are more often avoided compared to max - link. Also, the theoretical curve of the outage probability matches the simulation results validating the analysis in Section IV. As $L$ increases, the curves become steeper, thus indicating the increase in diversity as more links are available for selection.

Average Throughput. Next, we present average throughput comparisons. Fig.4 depicts the performance of each scheme. First, we see that the compared policies are divided in two groups. The first consists of the half-duplex schemes, namely BRS, max - max and max - link. Due to the constant transmission rate, equal to $1 \mathrm{bps} / \mathrm{Hz}$, these schemes can achieve a maximum average throughput of $0.5 \mathrm{bps} / \mathrm{Hz}$. In line with the outage probability performance, max - link outperforms BRS and max - max and reaches the upper bound nearly 2.5 $\mathrm{dB}$ prior to the others. In the second group we have SOR, BA-SOR and $\min$ - power. min - power achieves the best performance reaching $1 \mathrm{bps} / \mathrm{Hz}$ for high SNR. BA-SOR has a performance gap as it is not coupled with a robust single-link selection scheme while SOR does not reach the upper bound even for high SNR as IRI and the lack of buffering cause many outages. When $L$ increases, min - power follows the selection bound and their performance gap closes at about $8 \mathrm{~dB}$. It is important to note that when the SNR is low, interference cancellation does not take place often and the proposed scheme chooses half-duplex transmissions instead of successive ones.

\section{Vi. Conclusions And Future DiRections}

An opportunistic relaying protocol that minimizes the total energy expenditure per time slot under an IRI cancellation scheme is proposed. Making use of power adaptation and buffer-aided relays, the detrimental effect of IRI through interference cancellation is mitigated. We investigated the performance of the proposed relay-pair selection scheme in terms of outage probability and average throughput and comparisons with other state-of-the-art relay selection schemes were presented. The results indicate that the proposed min - power

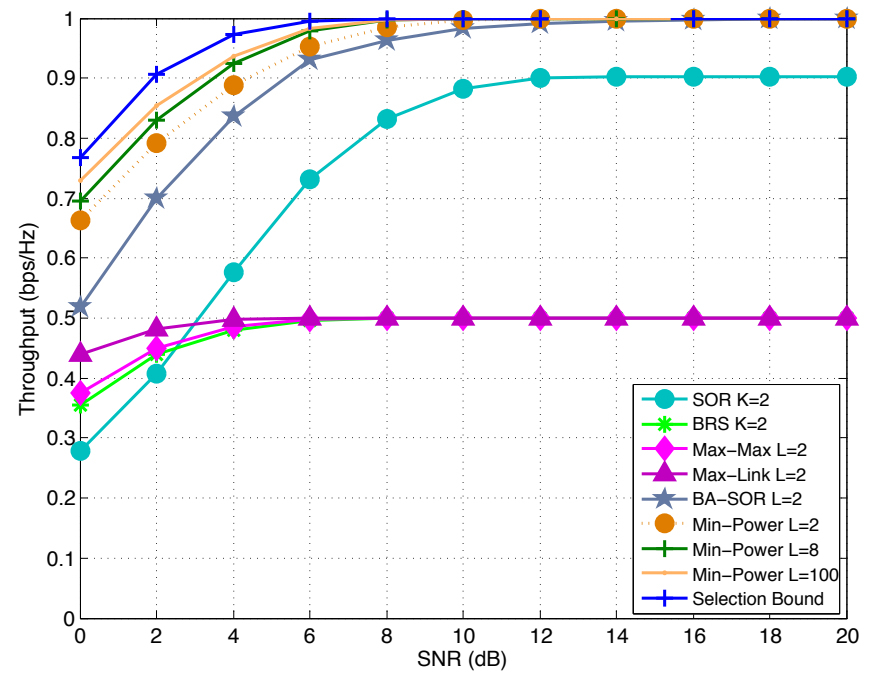

Fig. 4. Average throughput for increasing transmit SNR for $K=2$.

offers improved performance in terms of outage probability and average throughput.

When instantaneous CSI is available, power adaptation can be used to optimize the SINR. This approach, however, is not feasible when the channels change fast. It would be interesting to consider the case for which the channels change fast and only statistical channel information is available.

\section{REFERENCES}

[1] J. N. Laneman, D. N. C. Tse, and G. W. Wornell, "Cooperative diversity in wireless networks: Efficient protocols and outage behavior", IEEE Trans. Inform. Theory, vol. 50, pp. 3062-3080, Dec. 2004.

[2] Z. Ding, I. Krikidis, B. Rong, J. S. Thompson, C. Wang, and S. Yang, "On combating the half-duplex constraint in modern cooperative networks: protocols and techniques," IEEE Wireless Commun., vol. 19, pp. 20-27, Dec. 2012.

[3] Y. Fan, C. Wang, J. S. Thompson, and H. V. Poor, "Recovering multiplexing loss through successive relaying using repetition coding," IEEE Trans. Wireless Commun., vol. 6, pp. 4484-4493, Dec. 2007.

[4] A. Bletsas, A. Khisti, D. Reed, and A. Lippman, "A simple cooperative diversity method based on network path selection," IEEE J. Select. Areas Commun., vol. 24, pp. 659-672, March 2006.

[5] D. S. Michalopoulos and G. K. Karagiannidis, "Performance analysis of single relay selection in Rayleigh fading," IEEE Trans. Wireless Commun., vol. 7, pp. 3718-3724, Oct. 2008.

[6] I. Krikidis, J. S. Thompson, Steve McLaughlin and Norbert Goertz, "Max-min relay selection for legacy amplify-and-forward systems with interference," IEEE Trans Wireless Commun., vol. 8, no. 6, pp. 30163027, June 2009 .

[7] A. Ikhlef, D. S. Michalopoulos, and R. Schober, "Buffers improve the performance of relay selection," Submitted to IEEEE Global Commun. Conf., March 2011.

[8] A. Ikhlef, D. S. Michalopoulos, and R. Schober, "Max-max relay selection for relays with buffers," IEEE Trans. Wireless Commun., vol.11, no.3, pp.1124-1135, March 2012.

[9] A. Ikhlef, K. Junsu, and R. Schober, "Mimicking full-duplex relaying using half-duplex relays with buffers," IEEE Trans. Vehicular Tech., vol.61, no.7, pp.3025-3037, Sept. 2012.

[10] I. Krikidis, T. Charalambous, and J. S. Thompson, "Buffer-aided relay selection for cooperative diversity systems without delay constraints," IEEE Trans. Wireless Commun., vol.11, no.5, pp.1957-1967, May 2012.

[11] N. Nomikos, D. Vouyioukas, T. Charalambous, I. Krikidis, D. N. Skoutas, and M. Johansson, "Capacity improvement through buffer-aided successive opportunistic relaying," IEEE Wireless Vitae Conf, June 2013.

[12] N. Nomikos, and D. Vouyioukas, "A successive opportunistic relaying protocol with inter-relay interference mitigation," IEEE Wireless Commun. and Mob. Comp. Conf, pp. 228-233, August 2012. 